Aus der dritten medizinischen (Nerven-) Abteilung des Allgemeinen Krankenhauses Hamburg-Barmbeck.

\title{
Weiterer Beitrag zur Pathologie der Zirbeldrüse.
}

Von

\section{Prof. H. Iuce.}

In den letzten Dezennien hat das Interesse der Kliniker in stetig steigendem Maße der Erforschung des endokrinen Drüsensystems sich zugewandt. Eine respektable, durch ihre Mannigfaltigkeit und Fülle fast verwirrende Summe von klinischen, pathologischen und experimentellen Tatsachen ist durch emsige Arbeit fleißiger Forscher aus aller Herren Länder zusammengetragen worden, die Kompilation des also Gewonnenen hat ihren Niederschlag in zahlreichen kuihnen, oft nur allzu kühnen Hypothesen gefunden, die das charakteristische Zeichen des gegenwärtigen medizinischen Zeitalters, die humorale Denkweise, unverkennbar an der Stirn tragen. Die klinischen Erfahrungen haben die Erkenntnis gezeitigt, daß die einzelnen Hormonproduzenten im Organismus nicht als in sich unabhängige Betriebsorganisationen angeschaut werden können, vielmehr sind sie in ihren Beziehungen zueinander den Gesetzen der funktionellen Reziprozität unbedingt unterworfen.

Demgemäß wollen pathologische Abartungen hormonaler Funktionen unter dem Gesichtswinkel betrachtet sein, daß sie niemals eine positive oder negative Variante einer bestimmten Organtätigkeit allein zur Darstellung bringen, sondern da $\beta$ die im klinischen Bilde sich ausdriickende Dyshormonie ihrerseits als abgeändertes Produkt intraorganischer funktioneller Reziprozität im ganzen endokrinen System aufgefaßt werden muß.

Unser Wissen bezüglich des endokrinen Systems steht demgemäß durchaus im Zeichen und im Fluß gärender Erkenntnis. Mit Rücksicht auf die märchenhafte Kompliziertheit aller in Betracht kommenden Verhältnisse ist es nur zu begreiflich, daß einige Drüsen lange Zeit als stumme Organe imponieren konnten, bis ein glïcklicher Zufall dem 
ärztlichen Beobachter Licht in das Dunkel fallen ließ, das bis dahin das Geheimnis ihrer physiologischen Natur verhüllt hatte.

Dieses Los hat auch die Zirbeldrüse gehabt, welche erst vor gut einem Jahrzehnt durch eine ausgezeichnete Arbeit v. Frankl-Hochwarts ihrer Anonymität entkleidet werden zu sollen schien und der neurologischen Diagnostik zugänglich gemacht wurde. v. Frankl konnte auf Grund einer von ihm im Jahre 1909 bei einem Knaben gemachten Beobachtung den diagnostischen Satz formulieren: „Wenn bei einem sehr jugendlichen Individuum (Knabe) neben den allgemeinen Hirntumorsymptomen sowie dem Nothnagelschen Syndrom (nartielle (phthalmoplegie, statische Ataxie) abnormes Längenwachstum, ungewöhnlicher Haarwuchs, Adipositas, Sehlafsucht, vorzeitige Genital- und Sexualtätigkeit, allenfalls géistige Frühreife sich finden, dann hat man an eine Geschwulst der Zirbeldrüse (Teratom) zu denken."

Die klassische Beobachtung Frankls gab zunächst den Anstoß zum Ausbau der kasuistischen Krfahrungen über die Zirbelpathologie und regte den Japaner Sunji Uemura $\left.{ }^{1}\right) 1917$ und den Wiener Schlesinger ${ }^{2}$ ) 1918 zur pathologisch-anatomischen Neubearbeitung des kleinen Organs an, in welchem im 17. Jahrhundert der französische Philosoph Descartes den Sitz der Seele vermutet hatte. Uemura stellte fest, daß die Zirbel zwar einerseits histologische Zeichen widerspiegelt, die auf Gewebsabbau hindeuten, da $B$ sie aber andererseits - ebenfalls aus histologischen Gründen - ein Organ mit Funktion darstelle. Schlesinger kam auf Grund seiner Studien zu dem Urteil, da $B$ man nicht berechtigt sei, die Zirbel als ein nach der Pubertät scbwer degeneriertes, kaum funktionsfäliges Organ anzusprechen, sie sei vielmehr auch im späteren Alter ein wichtiges Gebilde mit Funktion, das vermutlich eine andere als eine rein dekorative Rolle spiele.

Es soll dahingestellt bleiben, ob gerade für die Zirbel rein histologische Kriterien ausreichend sind, um einem Organ funktionelle Daseinsberechtigung als aktive Blutdrüse zuzusprechen, dessen entwicklungsgeschichtliche Vergangenheit aus dem Dache des Zwischenhirns so ausgesprochene Hinweise auf seine Abstammung als Sinnesorgan, das berühmte Scheitelauge der Saurier, enthält. Es ist doch sehr wesentlich zu berücksichtigen, daß die bisher aus der menschlichen Pathologie vorliegenden Beobachtungen ausschließlich bei

1) Shunji Uemura, Frankf. Zeitschr. f. Pathol. 1917, Bd. 20.

2) Schlesinger, Neurol. Centralbl. 1918, S. 693. 
Gesehwulstprozessen der Zirbel gewonnen sind. daß durch eine solche Komplikation einmal die echte Natur der Zirbel bezüglich ihrer physiologischen Wertigkeit nur zu leicht verfälscht werden kann und daß andererseits die raumbeschränkend wirkenden Zirbelgeschwülste unvermeidlich mechanische Druckwirkungen ausüben müssen auf ihre Nachbarschaft (Vierhügel, 3. und 4. Ventrikel, Aquaeductus Sylvii, Zwischenhirn), und diese ist physiologisch von außerordentlich großer Bedeutsamkeit. Im Grunde genommen wissen wir über die ureigentliche Funktion der Zirbel zurzeit rein gar nichts, weil eben alle Erfahrungen aus der menschlichen Pathologie an Fällen mit geschwulstartiger Umwandlung des Organs gewonnen sind und weil die tierexperimentelle 7irbelforschung voll der gröbsten Widersprüche ist. Wie unsicher alle unsere Kenntnisse nach dieser Richtung sind, zeigt schlagend die Arbeit Hofstätters (Ergebnisse und Aussichten der experimentellen Zirbelforschung, Jahrb. f. Psych. u. Neurol. 1917, Bd. 37). Aus der Fülle der dort niedergelegten Tatsachen will ich nur einige wenige herausgreifen. Creutzfeld berichtete schon 1913 über das Fehlen der Epiphyse bei Säugetieren (Crocodilus, Dickhäuter, Wale, Gürteltiere), bzw. über hochgradige absolute und relative Unterentwicklung dieses Organs. Schon allein durch diese Beobachtung wird die Hypothese aufs schwerste erschuittert, derzufolge der Zirbel eine die sexuelle Entwicklung regulierende und hen̂mende Funktion zukommt. Gegenüber Foà kam Cristen bei 30 jungen Hähnen zu genau entgegengesetzten Ergebnissen: die operierten Tiere blieben nämlich zurück in der Entwicklung der sekundären Sexualcharaktere (Kamm, Bart, Sporen, Gesang), zeigten Hodenatrophie. Auch Foà fand später in neuen Versuchen an Hühnern bei den operierten Tieren keine Veränderung an den Testikeln, speziell keine der interstitiellen Drüse. Dandy beobachtete sowohl bei alten wie jungen Hunden, daß die Zirbelexstirpation ohne Einfluß auf die Genitalsphäre blieb. Nach Kastration fanden Biach und Hulles Atrophie der Zirbel, Pellegrini Hypertrophie von Zirbelzellen. Lindemann und Aschner fanden Epiglandol wehenerregend, Frankl-Hochwart konnte mit Epiglandol Roche am Uterus gravider Meerschweinchen Relaxation hervorrufen usw. Also soviel Tatsachen. soviel Widersprüche!

Aber auch die Pathologie der Zirbel des Menschen ist keineswegs frei von krassen Widersprüchen. Die Mehrzahl der mitgeteilten Beobachtungen über Zirbelgeschwülste, und zwar nicht nur der Teratome, sondern auch der Gliome und Sarkome ist klinisch charakterisiert 
durch Pubertas praecox (alle Beobachtungen betrafen Knaben). Im Gegensatz hierzu berichten Raymond und Cla ude (s. Ernst Boehm, Frankf. Zeitschr. f. Pathol. 1919, Bd. 22) über einen 10 jährigen Knaben mit den seit 3 Jahren bestehenden Allgemeinerseheinungen des Hirntumor mit Erblindung. Der Knabe war $138 \mathrm{~cm}$ groß, $39 \mathrm{~kg}$ schwer (normal nach Camerers Tabellen: 127,3 cm und 29,4 kg) Penis und Hoden waren klein, Bart und Schamhaare stark entwickelt. Allgemeine $\Lambda$ dipositas. Die Sektion ergab ein die Umgebung infiltrierendes Gliom der Zirbel. Die Nebennieren waren hyperplastisch. Im atrophischen Hoden fanden sich gut entwickelte Leydigsche Zwischenzellen.

Dieser Fall der französischen Autoren fällt vollständig aus dem Rahmen der übrigen Beobachtungen heraus. Während diese übereinstimmend - gleichgültig, ob Teratom, Gliom, Sarkom der Zirbel vorlag - Hypertrophie von Penis und Hoden in ihren Fällen notieren, berichten Raymond und Claude in ihrem Fall ausdrücklich, daß Penis und Hoden (trotz gutentwickelter Leydigscher Zellen) klein waren. Berücksichtigt man ferner den Umstand, daß Pubertas praecox bei Mädchen mit Zirbelgeschwïlsten bisher überhaupt nie beobachtet wurde, vielmehr bei solchen nur im Falle von Neoplasmen der Nebennieren, so steigert sich die Wahrscheinlichkeit, daß es nicht die geschwulstartige Umwandlung der Zirbel ist, welche durch Hyper-oder durch Hypo-Pinealismus oder durch Stoffwechselprodukte der Teratome (Askanazy) den Anstoß dazu abgibt, daß das Syndrom der Pubertas praecox in die Erscheinung tritt. Es dürfte vielmehr das vermittelnde Zwischenglied in der Schädigung oder Reizung zu suchen sein, welche der Zwischenhirnboden mit seinen lebenswichtigen sympathischen Zentren rein mechanisch, direkt und indirekt, durch die von seiten der Zirbelgeschwulst ausgeübten Druckwirkungen erleidet.

Außerdem ist es im konkreten Falle bei Zirbelgeschwülsten ausgeschlossen, festzustellen, welcher Anteil in der klinischen Symptomatologie auf Rechnung der Dysfunktion der spezifischen Parenchymzellen zu setzen ist und welcher Anteil auf Schädigung des unmittelbar benachbarten Zwischenhirnbodens mit seiner eminent hochwertigen physiologischen Beschaffenheit. In allen den klinisch und diagnostisch verwertbaren Fällen, in welchen die Kasuistik symptomatologische Hinweise auf die scheinbare Funktion der Zirbel enthält, lagen, wie gesagt, geschwulstartige Prozesse der Epiphyse vor mit entsprechender 
raumbeschränkender Tendenz, die selbstverständlich durch Einbeziehung indirekter Herdsymptome die Originalfunktion des Organs maskieren oder gar unterdrücken mußten.

Ich $^{1}$ ) habe bereits im Jahre 1914 auf meiner Abteilung den Fall erlebt, daß ein 5jähriges Mädchen mit den Erscheinungen der Hirngeschwulst zur Aufnahme gelangte, bei welchem die Gruppierung der Symptome (Nothnagelsches Syndrom in Verbindung mit leichter Adipositas) auf einen im Bereich des Zwischenhirndachs gelegenen raumbeschränkenden Prozeß hinwies. Die gewohnheitsgemä $\boldsymbol{\beta}$ vorgenommene Röntgenaufnahme des Schädels (Prof. Haenisch) brachte auf der Platte einen im Schädelinnern freischwebenden kleinen Kalkschatten zur Darstellung. Damit war für mich die Diagnose Zirbeldrüsengeschwulst sofort gegeben und konnte auch durch die bald darauf vollzogene Sektion vollauf bestätigt werden: Die Zirbel war in eine pflaumengroße, leicht höckrige Geschwulst umgewandelt und konnte Prof. Fahr in ihr die Elemente des Teratoms aus 3 Keimblättern und das Vorhandensein von Kalk mikroskopisch nachweisen. Ich glaubte daher, die diagnostische Formulierung Frankls dahin erweitern zu können, daß ich sagte: Die Diagnose einer Zirbelgeschwulst ist gesichert, wenn neben dem Nothnagelschen und neben dem Franklschen Syndrom bei einem jugendlichen Individuum der Nachweis eines umschriebenen Kalkschattens auf der Röntgenplatte erbracht werden kann. Dieser muß in seiner Topographie der Lage der Zirbel entsprechen.

Bekanntlich geht die pathologisch-anatomische Auffassung dahin, daß der Hirnsand in der Zirbel, in den Plexus chorioidei und in den Hirnhäuten bei vorgerücktem Lebensalter, in mikroskopischer Menge wenigstens, normal vorkommt, daß er in hyperplastischen und sarkomatösen Geschwiilsten dieser Hirnteile so massenhaft auftritt, daß davon die Bezeichnung Psammom (Sandgeschwulst) stammt (Recklinghausen). Dengemäß wird allein schon der röntgenologische Nachweis eines der Topographie nach der Zirbelgegend entsprechenden freischwebenden Kalkschattens im Schädelinnern den Schluß zulassen, daß die Epiphyse eine irgendwie geartete materielle Veränderung eingegangen ist. Seitdem ich meine Aufmerksamkeit diesem Verhalten der Zirbel zugewandt habe, hat sich bei mir der Findruck befestigt,

1) Deutsche Zeitschr. f. Nervenheilkunde Bd. 68/69. Zur Diagnostik der Zirbelgeschwülste und zur Kritik der cerebralen Adipositas. 
I daß der Zirbelkalkschatten, wenn er röntgenologisch darstellbar wird, auch stets eine gröbere pathologische Veränderung der Zirbel zur , Voraussetzung hat. Die schon vom 1. Lebensjahrzehnt ab in dem in beginnender Rückbildung begriffenen Organ mikroskopisch nachweisbaren Kalkspuren kommen naturgemä $B$ für die röntgenologische Erfassung derselben nicht in Betracht; dazu sind gröbere Kalkbeimengungen in dem Gewebe der Zirbel erforderlich, damit ihr Voihandensein auf der photographischen Platte fixiert werden kann.

Ein solcher von einer Kalk führenden Zirbel gelieferter Schatten präsentiert sich auf der Röntgenplatte als ein winziger, unregelmäßig gestalteter Fleck von 1-2 $\mathrm{nm}$ in Breiten- und Höhendurchmesser.

Soh üller ${ }^{1}$ ), der schon 1914 die Verkalkung in der Zirbel als die gewöhnliche intrakxanielle Verkalkung angesprochen hat, beschreibt die Topographie dieses Schattens folgendermaßen: Derselbe liegt $4,5 \mathrm{~cm}$ dorsal von der deutschen Horizontallinie und $1 \mathrm{~cm}$ hinter der Frontalebene durch den äußeren Gehörgang in der Mitte des Schädels. Damit ist dieser Zirbelkalkschatten topographisch genau festgelegt. Das ist aus dem Grunde wichtig, weil neuere röntgenologische Arbeiten von Heuer ${ }^{2}$ ), Dand $y^{2}$ ) und kürzlich von J.Ström ${ }^{3}$ ) den Nachweis erbracht haben, daß im Schädelinnem Kalkschatten von allen möglichen Lokalisationen, Formen und Größen zur Darstellung gebracht werden können, und zwar hat es sich dabei gehandelt um Verkalkungen, welche in Geschwülsten (Psammome, Endotheliome, Sarkome, Hypophysengangsgeschwülste, Gliome, Cysten, Tuberkel), aber auch in Aneurysmen, in encephalitischen Herden, nach Traumen, nach Blutungen und aus unbekannter Herkunft zur Beobachtung gekommen waren. Es wird Aufgabe des Klinikers sein, an der Hand einer eingehenden Analyse der Vorgeschichte und des Verlaufs der Krankheit zu entscheiden, in welchem Sinne die röntgenologisch also gewonnenen Feststellungen diagnostisch zu verwerten sind.

Die Fähigkeit der Zirbel, Kalk innerhalb ihres Gewebes festzuhalten und niederzuschlagen, ist nicht darauf zurückzuführen, daß etwa eine spezifische Affinität dieses Organs zu den im Blute kreisenden Kalksalzen vorhanden wäre, sondern sie ist ohne $Z_{\text {weifel in der früh- }}$

1) Schüller, Lewandowskys Handbuch der Neurol. 1914.

2) Heuer u. Dandy, Roentgenography in the localisation of brain tumour. Bulletin of St. John Hopkins Hospital 1916, p. 311.

3) Ström, Fortschr. Röntgenstrahlen, Bd. 27. 
zeitig einsetzenden und mit degenerierenden Gewebsveränderungr * verbundenen Rückbildung der Zirbel zu suchen. Hierbei kommt uzu einem Ersatz des spezifischen Drüsengewebes durch Gliaplatte. ${ }^{\text {ht }}$ die teils verkalken, teils erweichen und zu sekundären Cystenbilduns. ${ }^{\text {s- }}$ führen.

Nach meinen Erfahrungen wird diese Tendenz der Zirbel $z^{\text {:n }}$ Rückbildung, die in keinem zweiten Organ des Körpers so frühzeit ${ }^{3-}$ einsetzt wie hier, ungemein gefördert durch Traumen, welche $d^{s-}$ Schädel getroffen haben. Die Erschütterung, welche die Zirbel $k^{n-}$ einer Gewalteinwirkung auf den Schädel erfährt, führt zu molekular ${ }^{\text {LS- }}$ Schädigungen ihrer Gewebsstruktur und zu kleinsten intraglanduläriuBlutungen, wie solche ja mit Vorliebe bei der Hirnerschütterung itden um den Aquaeductus und um den 4. Ventrikel herum gelegenter Gewebspartien aufzutreten pflegen. Im Anschlu $B$ an solche Erschich terungen treten dann regressive Veränderungen in der Zirbel auf, ise ihrerseits eine Einlagerung von Kalk zur Folge haben.

er-

Ich habe im vorigen Jahre Gelegenheit gehabt, die Richtigk dieser Anschauung durch eine klinische Beobachtung, die hier foller bestätigt zu sehen. ich

13jähr. Engländer. Beide Eltern zucherkrank, Mutter basedowkraten Tadellose körperliche und geistige Entwicklung. Scharlach mit nacobfolgender Albuminurie, die ausheilte. Nie nervös, nie Konvulsionen. en-

Februar 1920, Schädeltrauma: Beim Rollschlittscbuhlaufen sch kel er mit dem Kopf gegen die Wand, für einige Minuten bewußtlos. Ap großes, dickes Hämatom über der rechten Scheitelgegend. Erbrecl en. das nach einem Tage sistierte. ale

Juli 1920 ohne Ursache Erbrechen, das bald täglich, sehr bald meEr-

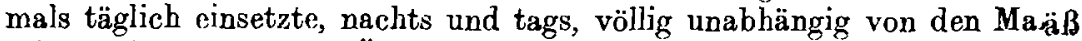
zeiten, ohne Vorboten (Utbelkeit, Schwindel). Wurde seit Juli auffalle lie fett und blieb im Wachstum zurück. Geistig von jeher sehr gut begabt ${ }_{21}$ Knabe.

Wilson (London) diagnostizierte Epilepsie und ordinierte Brom. th

Status 9. II. 1921: $138 \mathrm{~cm}$ großer, 39,5 kg schwerer Knabe. Kop:T umfang $55 \mathrm{~cm}$. Ausgesprochene allgemeine Fettleibigkeit, einschließlicif des Gesichtes. Besonders starke Entwicklung des Panniculus adiposts um Hüften, Gesäß, Mons veneris und Brust. Ausgesprochener weiblicher Gesamthabitus. Achselhöhlen, Mons pubis völlig haarlos. Volles Haupthaar. Kein Schnurrbart. Innere Organe gesund. Beide Hoden deutlich kleiner als dem Alter entsprechend. - Urin frei von Eiweiß und Zucker. Blut-Wassermann ø. Völlig nor maler Befund am Nervensystem. Augenhintergrund normal.

Röntgenaufnahme (Prof. Haenisch): Schädelaufnahme in rechter und linker Seitenlage. Sella turcica durchaus normal, keinerlei Erweite- 
g oder Abflachung. Etwas oberhalb und dorsal vom Os temporale der typischen Stelle ein kleiner unregelmäßiger Kalkschatten, der der send der Ziı beldrüse enstpricht.

17. II. 1. Lumbalpunktion ergibt: Liquordruck $=400 \mathrm{~mm}$; Liquor pft nur langsam ab, völlig klar, $10 \mathrm{ccm}$ abgelassen bis zum Druck von i mm; Zellen 10: 3; Nonne, Pandy, W eichbrodt $\emptyset$, ebenso Wassermann $\varnothing$.

Blutzucker 0,14\% (Dr. Feigl).

Die klinische Beobachtung der nächsten Wochen stellte fest, daß

Knabe in 24 Stunden 7-12 Anfälle hatte, die charakterisie:t waren ch Ubelkeit, Würgen - unabhängig von der Nahrungsaufnahme sei große Blässe. Alles dieses häufiger ve'gesellschaftet mit kurz dauern1 Absenzen. Die nachts auftretenden Anfälle waren velbunden mit ihlen im Bett, Würgen, selten mit Erbrechen, ohne daß Patient dabei vachte. Nie Einnässen oder Stublabgang ins Bett. - Puls bewegte $h$ dauernd zwischen 64 und 80.

4. III. 2. Lumbalpunktion: Druck $430 \mathrm{~mm}, \Lambda$ blassen von $200 \mathrm{ccm}$ i zu einem Druck von $220 \mathrm{~mm}$. Liquor-Wasse mann abe mals $\emptyset$.

10. III. Zweimal täglich 1 Tablette Jodtropon; Beginn mit Einbungen mit Ungt. Ciner 4.

11. IV. Beendigung der Hg-Kur. Hat im ganzen $96 \mathrm{~g}$ Ungt. ciner. kommen und gut ve:t agen. In den letzten 6 Tagen in 24 Stunden nur -5 der oben beschriebenen Anfälle. Sehr gutes Allgemeinbefinden. Nie opfschmel zen.

19. IV. 3. Lumbalpunktion: Druck $120 \mathrm{~mm}$ !

Jodtropon abgesetzt. Beginn mit Luminal-Kur (3mal tägl. 0,05).

23. IV. Abreise aufs Land.

15. V. Sehr gut e' holt. Täglich 1, höchstens 2 Anfälle.

Abreise nach England.

5. IX. 1921 Brief der Mutter: Knabe hat seit Ende Mai 14 Pfund

Gewicht abgenommen, wird immer magerer, obwohl er $\mathrm{nz}$ kolossal ißt. In de'selben Zeit ist er $3,5 \mathrm{~cm}$ gewachsen. Hat it 2 Monaten keinen Anfall mehr gehabt. Ist absolut wohl, gesund, ein hr aufgeweckter Schüler, badet täglich in Eastbourne und taucht. Die nfälle haben sich bald nach $A b$ eise von Deutschland verlozen, verliefen lietzt ohne $\mathrm{E}$ :brechen. Luminal seit August abgesetzt.

Meine zweite Beobachtung betrifft einen 42 jährigen Sergeanten:

Aufnahme im Baımbecker K ankenhaus 23. II. 1919. Am 29. XII. 18 Kopfst eifschuB; sofort allgemein ge'ähmt, nur rechter Arm etwas eweglich. Am 4. I. 1919 Operation Feldlazarett. Bald merkliche Bessrung.

Status zurzeit: Auf der Höhe des Scheitelbeins neben dem Haarwirbel ine knapp hühne-eigroße Wunde mit entsprechendem Defekt der Haut. us der Tiefe frische Granulationen; in der Wunde mehre'e in Abstoßung egriffene Knochensplitte chen. - Spastische Paraplegie der Beine mit ensibilitätsstörung, mit doppelseitigem Fußklonus und Steigerung der 'atellarreflexe. Ubriges Nervensystem o. B. 
Röntgenbild (Prof. Haenisch): „Ein Sequester im Schädeldefe] ist nicht nachweisbar. Als Nebenbefund Verkalkung in der Zirbeldrüse

Diese beiden Beobachtungen machen auf das Schönste anscha lich, wie Schädeltraumen mit nachfolgender Hirnerschütterung nic nur de unmittelbar um das zentrale Höhlengrau gelegenen Geweb partien in Mitleidenschaft ziehen, was ja längst bekannt ist, sonde: sogar ihre Erschütterungswelle bis in die Zirbel hinein auswirke lassen können. Die durch einen solchen Vorgang verursachte Geweb schädigung bewirkt Ausfällung von Blutkalksalzen in den in regre siver Veränderung befindlichen Partien der Zirbel. Diese wird nt mehr auf Grund der also herbeigeführten physikalischen Zustanc änderung der Darstellung und Festhaltung auf der Röntgenplatte gänglich. Ich möchte es für möglich halten. daß die infolge von $\mathrm{Bl}$ infiltration und Gewebsreaktion zunächst geschwollene und spä dureh ihren Kalkgehalt schwerer gewordene Zirbel rein mechanis als Ventil auf die dünne Vierhïgelplatte drücken und auf diese We den Abfluß des Liquors aus dem 3. Ventrikel in den Aquädukt schweren und den $Z$ wischenhirnboden reizen kann.

Die Berechtigung au einer solchen Annahme ergibt sich aus Erfahrung, daß Zirbelgeschwïlste $\ldots$ deneu allerdings wesentl größere Ausmaße zustehen als es bei der rein traumatisch veränder Zirbel der Fall sein dürfte - in der überwiegenden Zahl der Be achtungen durch starken Hydrocephalus der Seiten- und des 3. V trikels ausgezeichnet waren, während der $A c_{q} u a ̈ d u k t$ und der t.Ventri normale Kaliberverhältnisse in solchen Fällen aufzuweisen pflegt Es darf allerdings nicht vergessen werden. daß es ja das ganze zentr Höhlengrau ist, welches teils molekularer, teils hydrostatischer schütterung durch das Trauma ausgesetzt wurde, da $B$ demgem der nach einem solchen Trauma auftretende Hydrocephalus nur Reaktion des Ependyms auf das zur Einwirkung gelangte Schäd - trauma darstellt. Aber denkbar ist es sehr wohl, daß die traumatis zunächst vergrößerte und später durch Kalkinkrustation schwer gewordene Zirbel ihrerseits auch mechanische Druckwirkungen au die Vierhügelplatte ausübt. Die klinische Beobachtung, da $B$ in meinem ersten Falle trotz hohen Uberdruckes der Liquor auffallend langsam, Tropfen für Tropfen abströmte, so daß man geradezu den Eindruck hatte, daß jeder einzelne Tropfen sich mühsam herausquälen müsse - obwohl doch erfahrungsgenäß bei freier Passage der unter hohem Uberdruck stehenden Hirnkammern der Liquor aus der Tumbal- 
punktionsnadel hervorzuspritzen pflegt - diese Beobgchtung scheint mir dafür zu sprechen, daß die in den Seitenventrikeln gestaute Flüssigkeit nur mit Schwierigkeiten den AbfluB in den 4. Ventrikel sich verschaffen konnte, weil eben der Zugang zum Aquädukt durch das vergrößerte Gewicht der Zirbel relativ gesperrt wurde. Auch die schlieBlich eingetretene Ausheilung des Hydrocephalus nit Wiederherstellung normaler hydrostatischer Druckverhältnisse im Kammersystem würde in gewissem Sinne für eine solche Auffassung verwertet werden können. Es erscheint möglich, daß die durch arzneiliche Behandlung $(\mathrm{Hg}+\mathrm{K})$ angeregte und herbeigeführte Resorption des schädlichen Uberschusses an Flüssigleit in den Hirnkammern nicht nur die im Ventrikelependym vorhandenen materiellen Veränderungen, sondern ebenso auch gleichwertige Schäden in der traumatisch veränderten Zirbel zur Rückbildung bringen konnte. Der Erfolg war, daß die Zirbel auf ihren alten Lmfang, wenn nicht auf einen verringerten infolge von traumatiseher Atrophie sich einstellte. Damit war dann die Verkehrsstörung in den Kammerräumen endguiltig beseitigt, wie das durch das Ergebnis der 3. Lumbalpunktion bestätigt. wurde.

$\mathrm{Da} B$ die bezüglich des 1 . Falles vorgetragene Anschauung nicht nur rein theoretisches Postulat ist, das mag der Fall Gierkes bezeugen, bei welchem die krebsig vergrößerte Zirbel einer 72 jährigen Frau neben allgemeinen Reizerscheinungen als einziges Symptom einen Diabetes mellitus hatte in Erscheinung treten lassen.

Der bei meinem ersten Fall erhöhte Blutzuckergehalt $(0,14 \%)$ findet seine Erklärung wohl in der durch das Schädeltrauma bewirkten Schädigung des Zwischenhirnbodens und des zentralen Höhlengraus sowie in einer mechanischen Beeinflussung dieser hochwertigen Zentralstellen durch die traumatisch vergrößerte und verkalkte Zirbel.

Bei dem von mir beobachteten Patienten dürfte die Symptomentrias: Adipositas, Zurückbleiben im Wachstum, Hypogenitalismus, trotz röntgenologisch festgestellter Schädigung der Zirbel mit diesem Organ nichts zu schaffen haben, vielmehr als Ausdruck traumatischer Schädigung des Zwischenhirnbodens, des Trichters und der Hypophyse anzusehen sein. Der erhöhte Blutzuckergehalt beweist die Schädigung des Zwischenhirnbodens, die Schädigung der Hypophyse wird bewiesen durch das Zurïckbleiben im Wachstum und möglicherweise den Infantilismus der: Genitalorgane, obwohl ohne weiteres zuzugeben ist, daß letzterer keineswegs in Beziehung zum Hirnanhang zu stehen 
braucht. Es geqügt, daß ein primärer genitaler Infantilismus ursprünglich vorhanden war, der schon lange Zeit vor dem Trauma bestanden hatte. Es ist in höchstem Maße interessant zu verfolgen, wie mit der durch die Resorptionstherapie $(\mathrm{Hg}+\mathrm{K}$ ) angeregten Rückbildung der durch das Trauma im Umkreis des 3. Ventrikels und des Hirnanhangs hervorgerufenen Schäden schrittweise im Laufe eines halben Jahres die Adipositas abnimmt und das Längenwachstum zunimmt. Ich habe in meiner frïheren Arbeit den Standpunkt vertreten, daß es eine hypound epiphysäre Adipositas in dem Sinne, daß die Hormone dieser Drüsen einen Anreiz auf das Fettwachstum ausüben, nicht gibt, da $B$ vielmehr der Hydrocephalus allein an sich genügt, um die sympathischen Zentren im Bereich des Zwischenhirnbodens zur Mehrleistung anzuregen, und ich möchte glauben, daß diese Hypothese eine Stïtze findet in dieser neuen Beobachtung. Durch einen glücklichen Zufall war hier durch ein Schädeltrauma sowohl der Zwischenhirnboden als auch die Hypo- und Epiphyse schwer geschädigt worden, immerhin in einem Umfang, welcher die Wiederherstellung und Gesundung dieser traumatisch geschädigten Drüsen und des Ventrikelbodens nach Ablauf eines gewissen Zeitraumes zustandekommen ließ; allerdings nahm die traumatische Beeinträchtigung der beiden Drüsen einen grundverschiedenen Verlauf: Die Zirbel wurde geschädigt, aber ihre Funktion blieb anscheinend unversehrt, die Hypophyse wurde ernster in Mitleidenschaft gezogen und verriet in dem Zurückbleiben im Wachstum und möglicherweise in dem genitalen Infantilismus ihre Leistungsschwäche. Aus den einzelnen klinischen Phasen meines Falles läßt sich der sich hierbei abspielende biologische Vorgang in seinen Einzelheiten sehr gut verfolgen und ablesen: Geraume Zeit nach dem Trauma kam es infolge von Hypophyseninsuffizienz zum Wachstumsstillstand und allenfalls zum Hypogenitalismus, infolge von Hydrocephalus zur Entwicklung von Fettsucht; geraume Zeit nach dem Verschwinden des Hydrocephalus und nach Beendigung der Resorptionskur kam es zur Abnahme des Körpergewichts infolge von Schwinden des Hydrocephalus, kam es zum Wiedereinsetzen des Wachstums durch Wiederherstellung der Funktion der Hypophyse sowie infolge von Erholung des Zwischenhirnbodens. Der Zufall wollte, daß ich vor wenigen Wochen Gelegenheit hatte, einen zweiten Knaben von genau dem gleichen Alter zu beobachten, der ein schweres Schädeltrauma durchgemacht hatte, aber ohne Beschädigung der Zirbel. 
E. T. 13 Jahre alt aus Südbrasilien Porto Alegre. Stets gesund, geistig und körperlich ausgezeichnet entwickelt. Nie Krämpfe. 10. I. 1922 Sturz vom Baum $2 \mathrm{~m}$ tief. 3 Stunden bewußtlos. Hämatom am Okziput. Schwerer Schock mit Collaps. Keine Blutung aus Nase, Mund oder Ohren. Beiderseits Mydriasis mit träger Lichtreaktion, Cheyne-Stokes, später Bradykardie 60. 2 Stunden post trauma klonische Zuckungen rechte Hand, dann Gesicht, Hals und übrige Körperhälfte, schließlich auf die linke übergreifend. Daraus entwickelte sich ein status epilepticus. 1/2 Stunde nach Beginn der Krämpfe Lumbalpunktion: Liquor stark blutig, langsam abfließend, keine Drucksteigerung, $15-20 \mathrm{ccm}$ werden abgelassen. Nach Beendigung derselben noch ein Anfall, dann Erlöschen derselben. In den nächsten Tagen Erregung abwechselnd mit Apathie. Am 2. Tage post trauma erhebliche Besse: ung, kann wieder lesen, erkennt Umgebung. Vorübergehend lallende Sprache mit Echolalie. Ein paarmal retentio urinae. In der Genesung noch einige Zeit Fnuresis nocturna. Bald sehr gute Erholung und auffallend erhebliche Gewichtszunahme. Vollständige restitutio ad integrum nach 4 Wochen. Es besteht völlige Amnesie für die Zeit des Unfalls. Den klinischen Bericht verdanke ich der Güte der deutschen Ärzte Dr. Kühl und Dr. Richter.

12. VI. 1922. Aufgeweckter, dem Anschein nach blühend gesunder Knabe. G: ̈̈ße $125 \mathrm{~cm}$, Gewicht $44 \mathrm{~kg}$, Alter $13 \mathrm{Jahre.} \mathrm{(Camerers} \mathrm{Tabellen,}$ 13jähr. Knabe, Länge 140,6; Gewicht 38,2 kg.) Frische Blutfarbe der Schleimhäute. Auffallend stark entwickeltes Fettpolster im Bereich der Hüften, Nates, des Mons veneris, des Unterbauches und der Brustgegenden. Gesamthabitus durchaus feminin. Beide 'Testes in scroto, aber nur haselnußkerngroß! Absolute Atrichie des Kinns, der Lippen, der Áchseln, des Mons veneris. Stimme noch nicht gewechselt, kindlich. Innere Organe, Nervensystem völlig normale Verhältnisse.

Die von Prof. Haenisch vorgenommene Röntgenuntersuchung des Schädels konnte in der Zirbel keine Kalkdepots nachweisen. Dieser Knabe hat wohl zweifelsohne einen Schädelbruch mit schwerer Commotio cerebri und Blutung in den subarachnoidealen Raum durchgemacht. Die Zirbel blieb mit größter Wahrscheinlichkeit verschont, da im Röntgenbild Kalk in ihrem Gewebe nicht nachgewiesen werden konnte. Aber der Zwischenhirnboden, inkl. Hypophyse dürfte in Mitleidenschaft gezogen worden sein, sei es direkt oder indirekt durch Vermittlung eines Hydrocephalus. Das Längenwachstum betrug $125 \mathrm{~cm}$ gegeniiber $140,6 \mathrm{~cm}$ im Durchschnitt (beide Eltern sind große stattliche Menschen), das Gewicht $44 \mathrm{~kg}$ gegenüber $38,2 \mathrm{im}$ Dur'chschnitt. Die Mutter gab bestimmt an, bald nach dem Unfall sei eine sehr erhebliche Gewichtszunahme bei dem Knaben bemerkbar geworden. Die relative Kleinheit des Jungen ist in diesem Fall, wenn sie nicht schon vorher vorhanden war, zentralsympathischen bzw. 
hypophysären Ursprungs und auf eine traumatische Schädigung der Hypophyse zurückzuführen. Die Adipositas ist hier unbedingt cerebralen Ursprungs. Zufälligerwejse zeigte der kleine Patient eine hochgradige angeborene Hypoplasie beider Hoden (13 Jahre! Testes von der Größe eines Haselnußkerns!), auf welche die Eltern auch schon von ihren Ärzten in Brasilien aufmerksam gemacht waren, sein ganzer Habitus war weiblich, zeigte ausgesprochen genitalen Infantilismus. Es ist selbstverständlich, daß die traumatische Hypophysenschädigung für den genitalen Infantilismus nicht verantwortlich gemacht werden kann. Frfahrungen, wie diese sind sehr geeignet, eindringlich zu warnen vor vorzeitigen Verallgemeinerungen in bezug auf die innerhalb des endokrinen Systems vorhandene, aber noch stark aufklärungsbedürftige Reziprozität.

Den uns in meiner ersten Beobachtung gewordenen Einblick in die funktionellen Verhältnisse der tief im Schädelinnern gelegenen kleinen Drïse des Großhirns verdanken wir zweifelsohne den Fortschritten der hochentwickelten Röntgentechnik, welche es erlaubt, kleinste materielle Schädigungen der Zirbel der Beobachtung zugänglich zu machen und der ärztlichen Kontrolle zu unterwerfen. Der weiteren Forschung muß es vorbehalten bleiben, festzustellen, ob wir berechtigt sind, den Befund von röntgenologisch nachweisbarem Kalk in der Zirbel allemal als Zeichen pathologischer Vorgänge in dieser Drüse aufzufassen. Es sei nochmals betont, das wir nicht vergessen dürfen, da. $\beta$ schon unter physiologischen Verhältnissen gerade Zirbel und Plexus chorioideus es sind, welche Kalkdepots beherbergen, aber einstweilen liegen noch keine Erfahrungen darïber vor, da $B$ bereits die physiologischen Kalkeinlagerungen, die mit dem ersten Finsetzen der Involution schon im 4.-6. Lebensjahre anatomisch zu konstatieren sind, auch der röntgenologischen Darstellung zugänglich sind. Nach meinen bisherigen Beobachtungen möchte ich das für die ersten beiden Lebensjahrzehnte nicht für wahrscheinlich halten, vielmehr glauben, daß es hierfür pathologischer Kalkanreicherung bedarf, wenn der Zirbelkalk auf der Röntgenplatte darstellbar werden soll. Diese Conditio sine qua non dürfte vermutlich erfüllt sein, wenn eine Úmwandlung der Zirbel in Geschwulstgewebe stattgefunden hat. Das ist bei Fibromen, Sarkomen, Fndotheliomen, Teratomen dieses Organs der Fall, zu deren Entartungsform bekanntlich auch die Verkalkung gehört, ebenso dann, wenn eine traumatische Gewebsveränderung der Zirbel eingetreten ist, wie in den beiden von mir oben mitgeteilten Fällen. Unter solchen 
Umständen werden voraussichtlich die Bedingungen erfüllt sein, die eine gesteigerte Imprägnation des kleinen Organs mit Kalk gestatten, so daß nunmehr die röntgenographische Festhaltung der Kalkinkrustation auf der Platte ermöglicht wird. Die Lokalisation dieses Kalks auf dem Diapositiv wird ohne weiteres den Fingerzeig geben, in welchen Hirnteil diese Ablagerung zu verlegen sein dürfte. Die entsprechenden diagnostischen Schlußfolgerungen ergeben sich von selbst.

Aus meinen Darlegungen ziehe jch nunmehr folgende Schlïsse:

1. Schädeltraumen können auch die Zirbel in Mitleidenschaft ziehen.

2. Traumatische Zirbelschädigungen können der röntgenographischen Darstellung zugänglich werden, wenn vermehrte Kalkablagerung im Gewebe der Zirbel stattgefunden hat.

3. Traumatische Zirbelschädigung läßt das Syndrom der Pubertas praecox im 1. und 2. Iebensjahrzehnt nicht zustande kommen.

4. Traumatische Schädigung der Zirbel ist wohl meistenteils vergesellschaftet mit traumatischer Schädigung des Zwischenhirnbodens und der Hypophyse, aber das Umgekehrte braucht keineswegs der Fall zu sein.

5. Die traumatische Schädigung des Zwischenhirns bzw. der Hypophyse wird charakterisiert durch Adipositas, Zurückbleiben im Wachstum, Hyperglykämie. Aber das gilt nur für die ersten beiden luebensdezennien.

\section{Nachtrag.}

Im September 1922 stellte sich der kleine Patient (Beob. 1) mir wieder vor. Ausgezeichnetes Befinden. Dauernd frei von Anfällen, nur im Anschluß an Masern Januar 1922 zwei Anfälle. Seitdem nicht wieder. Der äußere Habitus ist völlig verändert, ausgesprochen männlich. Adipositas ganz verschwunden, Pat. ist mager, muskulös. Größe $152 \mathrm{~cm}$, also $+14 \mathrm{~cm}$ in 19 Monaten, Körpergewicht 42 Kilo. Hoden mehr als wallnußgroß, $4 \mathrm{~cm}$ lang, membrum $6 \mathrm{~cm}$ lang. Die sekundären Geschlechtscharaktere (Stimme, Haarkleid) noch nicht entwickelt. 